\title{
DISTRIBUCIÓN Y ESTADO DE CONSERVACIÓN DEL GÉNERO Haageocereus (FAMILIA CACTACEAE) EN EL DEPARTAMENTO DE LIMA. PERÚ
}

\section{DISTRIBUTION AND STATE OF CONSERVATION OF THE GENUS Hageocereus (CACTACEAE FAMILY) IN THE DEPARTMENT OF LIMA. PERU}

\author{
Natalia Calderón Moya-Méndez ${ }^{1}$, Aldo Ceroni Stuva ${ }^{1}$ y Carlos Ostolaza Nano ${ }^{2}$
}

\section{Resumen}

Se estudió la distribución y estado de conservación del género Haageocereus en el Departamento de Lima (Perú). Las plantas fueron recolectadas en 12 localidades ubicadas en las provincias de Canta, Huaral, Huaura, Huarochirí, Lima y Oyón, entre los 180 y 2500 msnm. El número de taxa evaluados fue un total de 12: 4 especies, 6 subespecies y 2 híbridos además de 2 formas, encontrándose en las siguientes categorías de conservación: Vulnerable (3), En Peligro (5) y En Peligro Crítico (6). La especie con menor distribución fue $H$. tenuis (Ritter) Rauh \& Ostolaza, la cual, por la alta vulnerabilidad de su único hábitat se ha incluido en la categoría de "En Peligro Crítico", mientras que la especie con mayor distribución fue $H$. acranthus (Vaupel) Backeberg, incluida en la categoría de "Vulnerable". Las localidades más seriamente perturbadas son Cajamarquilla, Santa Clara y camino a Huacho (km 118 Pan Norte), sugiriéndose la ejecución de planes de conservación ex situ para las especies $H$. pseudomelanostele (Werdermann \& Backeberg) Backeberg y H. tenuis (Ritter) Rauh \& Ostolaza.

Palabras clave: Cactus, distribución, conservación, categorización, género.

\begin{abstract}
Haageocereus genus distribution and conservation status in the Department of Lima (Perú) was studied. Plants were collected in 12 places located in Canta, Huaral, Huaura, Huarochirí, Lima and Oyón provinces, from 180 up to 2500 masl. The number of taxa evaluated was 12: 4 species, 6 subspecies and 2 hybrids, and also 2 forms, which were classified into the following conservation categories: Vulnerable (3), Endangered (5) and Critically Endangered (6). The lowest distribution species was $H$. tenuis (Ritter) Rauh \& Ostolaza, which has been included into the "Critically Endangered" category because of the high vulnerability of its sole habitat. On the other side, the highest distribution species was H. acranthus (Vaupel) Backeberg, categorized as "Vulnerable". Disturbed localities are Cajamarquilla, Santa Clara and on the way to Huacho (118 km North Panamerican Highway), where ex situ conservation plans for $H$. pseudomelanostele (Werdermann \& Backeberg) Backeberg and H. tenuis (Ritter) Rauh \& Ostolaza species are expected to be undertaken.
\end{abstract}

Key words: Cactus, distribution, conservation, categorization, genus.

\section{Introducción}

La familia Cactaceae en el Perú está representada por 34 géneros y más de 255 especies (Hunt, 1999), las cuales están distribuidas en casi todos los ecosistemas del Perú, desde los desiertos costeros, pasando por los valles interandinos, hasta la puna y llegando exitosamente al bosque tropical de nuestra amazonía, por lo que se puede hablar de una gran diversidad de adaptaciones de esta familia. Esta diversidad hace posible que el Perú albergue una considerable tasa de géneros y especies endémicas dentro de las cuales el género Haageocereus está bien representado. El género Haageocereus agrupa especies en su mayoría endémicas de nuestro país, las cuales se encuentran constituyendo diferentes formaciones vegetales caracterizando varios tipos de paisajes, como son el piso de cactáceas columnares de la vertiente occidental de los Andes y los desiertos propios de la Costa del Perú (Weberbauer, 1945). Su rango de distribución se extiende desde el Norte del Perú hasta el Norte de Chile a altitudes entre los 100 y $2500 \mathrm{msnm}$. Los hábitats naturales de las cactáceas están fuertemente perturbados por la expansión urbana, la contaminación ambiental, la creciente demanda de áreas de cultivo y pastoreo de ganado caprino (Ostolaza, 1988). En consecuencia, la categorización de las plantas, según su amenaza de extinción, es de vital importancia para llevar a cabo planes de conservación. Dicha categorización es efectuada por la Unión Internacional para la Conservación de la Naturaleza (UICN, 2001) utilizando básicamente dos criterios: la abundancia de un taxón (número de individuos) y el área total de ocupación de dicho taxón. El conocimiento de las especies y hábitats de Haageocereus ha sido posible por las expediciones que principalmente investigadores y comerciantes europeos realizaron durante el siglo XX. Destacan hacia 1919, los 
botánicos N.L. Britton \& J. N. Rose con su obra "The Cactaceae" (Britton \& Rose, 1919-1922), Curt Backeberg, quien en 1934 creó el género Haageocereus para incluir el grupo de plantas que Rose clasificó dentro del género Binghamia. Posteriormente Backeberg publicó su gran obra "Die Cactaceae" (Backeberg, 1960) entre 1959 y 1963, paralelamente y a veces en coautoría con Werner Rauh (1958). Importantes aportes al estudio de este género lo constituyen catálogos y descripciones de John Akers (1947), Ritter (1981), Brako \& Zarucchi (1993), Eggli \& Taylor (1990), Ostolaza (1980, 1988, 1995, 1996, 1998, 2002, 2003) y Calderón (2003). El presente trabajo tuvo como objetivo actualizar y difundir el conocimiento sobre la distribución y estado de conservación del género Haageocereus, como elemento importante de diversos ecosistemas del Perú.

\section{Materiales y métodos Ubicación}

El área de estudio corresponde a 12 localidades ubicadas en los valles de Huaura, Chillón, Rímac, Lurín y Santa Eulalia, en las provincias de Huaura, Huaral, Huarochirí, Canta, Lima y Oyón (Figura 1).

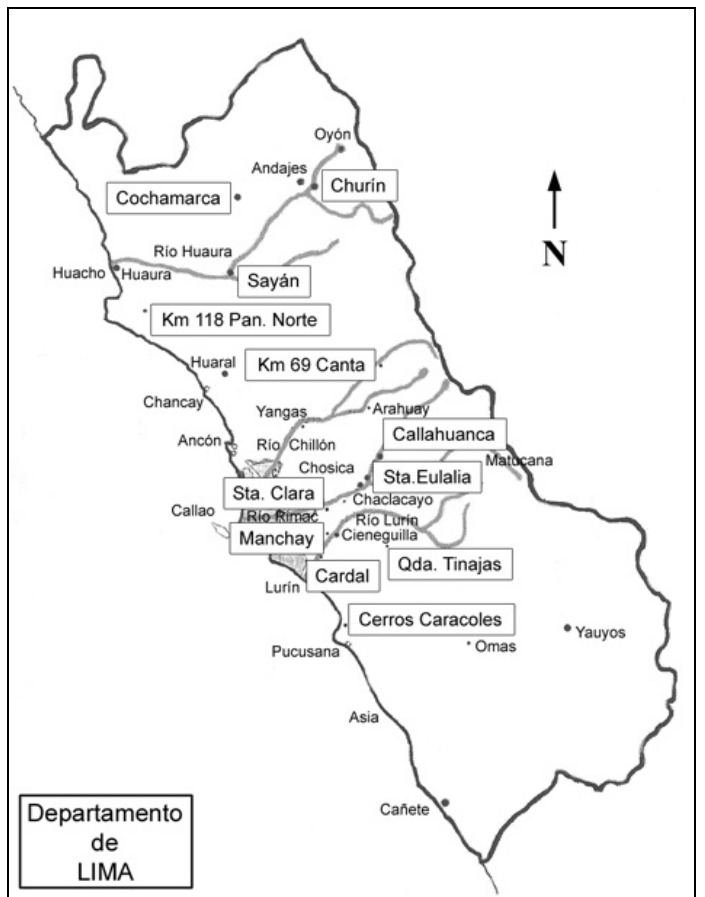

Figura 1. Localidades de colecta en el área de estudio.

\section{Caracterización de la zona de estudio:}

Área. Se seleccionó el área de colecta según la presencia de individuos que representen mejor las estructuras vegetativas y reproductivas de cada taxa en estudio.

Clima. Se puede establecer un rango térmico y de precipitación para todas las localidades en estudio. La temperatura media anual varía de $10.9^{\circ} \mathrm{C}$ en las zonas de mayor altitud, a $22.3^{\circ} \mathrm{C}$ en los matorrales desérticos de la costa. La precipitación es inversamente proporcional a la temperatura, mientras que la media anual es $711 \mathrm{~mm}$ para las zonas de mayor altitud, en las localidades próximas a la costa no llega a los 100 mm anuales.

Suelos. Los suelos son muy heterogéneos, desde arenosos hasta suelos de morfología netamente estratificada, de origen aluviónico y textura variadas. En las localidades próximas a la franja litoral dominan las arenas profundas, donde aparecen los regosoles. Se presentan también con cementaciones salinas, cálcicas o gípsicas con escasa materia orgánica, las formaciones líticas son típicas de las áreas empinadas. A lo largo de los valles aparecen los fluvisoles, de morfología estratificada, estos suelos son por lo general profundos, pudiendo ser muy arcillosos. En los valles occidentales los suelos son de textura media a pesada, de buen drenaje y en condiciones topofisiográficas empinadas, irrumpe la roca viva; se presentan también suelos calcáreos y gípsicos, además de andosoles (materiales volcánicos).

Fisiografía. El relieve se presenta plano u ondulado en la franja costera, variando a abrupto en los cerros aislados de la Costa. Hacia los valles se hace inclinado a empinado, desde suave a colinado. En los valles altoandinos se presentan terrazas, así como quebradas de pendientes pronunciadas.

Ecología. De acuerdo al Mapa ecológico del Perú (INRENA, 1995), el área de estudio presenta las siguientes zonas de vida: bosque seco-Montano Bajo Tropical (bs-MBT), desierto perárido-Montano Bajo Tropical (dp-MBT), desierto perárido-Premontano Tropical (dp-PT), desierto superárido-Sub Tropical (ds-S), matorral desértico- Premontano Tropical (mdPT), desierto desecado-Subtropical (dd-S) y matorral desértico- Montano Bajo Tropical (md-MBT).

\section{Metodología}

Recolección botánica. Se colectaron las especies del género Haageocereus en 12 localidades del Departamento de Lima (Figura 1), registrándose en etiquetas de campo los datos referentes a sus características, lugar de colección, altitud, etc. Para una adecuada colección, preparación y manejo de los especímenes, se siguió la metodología sugerida por Arakaki (2000) para cactus con tallos cilíndricos. Las plantas fueron colectadas entre los meses de Enero a Marzo en las localidades ubicadas en Caracoles y los valles de Lurín, Rímac, Chillón, Santa Eulalia y Chancay. Mientras que el valle de Huaura fue escenario de colectas y visitas en los meses de Junio y Agosto. Las altitudes de colección oscilaron entre los 180 y los 2500 msnm aproximadamente.

Categorización. Se tomó nota del estado de conservación según los reportes de Ostolaza (1995, 1996, 2002, 2003) y Ostolaza et al. (2003) para estas especies, el cual sigue la clasificación sugerida por la UICN (Unión Internacional para la Conservación de la 
Naturaleza) en su "Lista Roja" versión 3.1 Año 2001. Los criterios y categorías para las especies según la amenaza de extinción están clasificados en 9 categorías (Figura 2), las mismas que se mencionan a continuación: EXTINCT (EX): Extinto; EXTINCT IN THE WILD (EW): Extinto en su estado Silvestre; CRITICALLY ENDANGERED (CR): En Peligro Crítico; ENDANGERED (EN): En Peligro; VULNERABLE (VU): Vulnerable; NEAR THREATENED (NT): Cerca de Amenaza; LEAST CONCERN (LC): Menor Preocupación; DATA DEFICIENT (DD): Datos Deficientes y NOT EVALUATED (NE): No Evaluado. Como en previas Categorías de la UICN, la abreviación de cada categoría (en paréntesis) sigue las denominaciones inglesas cuando se traducen en otros idiomas, esto con fines de uniformizar la información para una gestión adecuada de los planes y acciones de conservación.

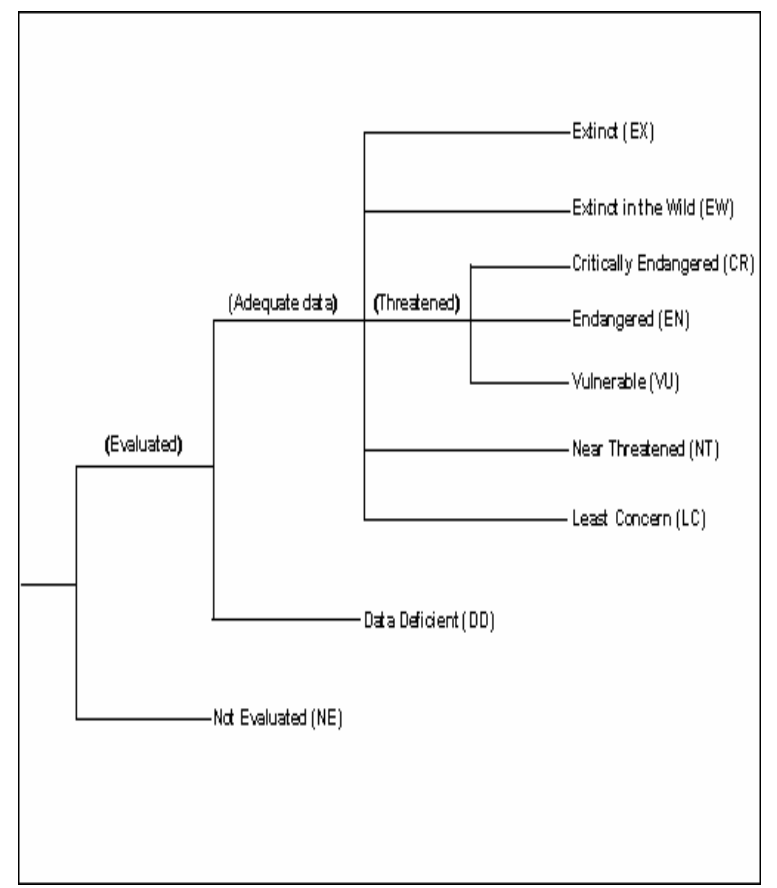

Figura 2. Estructura de las Categorías de Conservación (UICN, 2001. Ver. 3.1 Red List).

\section{Resultados y discusión}

Especies estudiadas. Los taxones correspondientes al género Haageocereus, colectados en el departamento de Lima fueron un total de 12: 4 especies, 6 subespecies y 2 híbridos, además de 2 formas (Tabla 1). La mejor época de colecta para material reproductivo de Haageocereus comprenden los meses entre Enero y Marzo.

Tabla 1. Especies, subespecies, formas e híbridos del género Haageocereus en el departamento de Lima. Perú.

\begin{tabular}{|c|l|l|}
\hline ESPECIE & SUBESPECIE & \multicolumn{1}{|c|}{ FORMA } \\
\hline Haageocereus & olowinskianus & clavispinus \\
\hline
\end{tabular}

\begin{tabular}{|c|c|c|}
\hline \multirow[t]{3}{*}{ acranthus } & & rubriflorior \\
\hline & deflexispinus & \\
\hline & Zonatus & \\
\hline \multirow{3}{*}{$\begin{array}{l}\text { Haageocereus } \\
\text { pseudomelanostele }\end{array}$} & acanthocladus & \\
\hline & carminiflorus & \\
\hline & Setosus & \\
\hline \multicolumn{3}{|l|}{$\begin{array}{l}\text { Haageocereus } \\
\text { albispinus }\end{array}$} \\
\hline \multicolumn{3}{|l|}{ Haageocereus tenuis } \\
\hline \multicolumn{3}{|l|}{$\begin{array}{c}\text { HÍBRIDOS } \\
\text { (Haageocereus } x \\
\text { Espostoa) }\end{array}$} \\
\hline \multicolumn{3}{|l|}{$\begin{array}{l}\text { XHaagespostoa } \\
\text { albisetata }\end{array}$} \\
\hline $\begin{array}{l}\text { XHaagespostoa } \\
\text { climaxantha }\end{array}$ & & \\
\hline
\end{tabular}

Distribución, categorización y estado de conservación

Haageocereus acranthus (Vaupel) Backeberg

Distribución: LIMA: Valle del Rímac, km 54 LimaLa Oroya, 1800 msnm; Valle del Chillón, km 64 Lima-Canta, $1260 \mathrm{msnm}$; Callahuanca - Valle de Santa Eulalia, 1300 msnm; Localidad cercana a Canta, 2700-2800 msnm; Prov. Lima, km 70 Carretera Central, 2200 msnm.

Categorización y Conservación: Según Ostolaza (2003) este taxón está en la categoría de "Vulnerable" (Vulnerable).

Haageocereus acranthus ssp. zonatus (Rauh \& Backeberg) Ostolaza

Distribución: LIMA; Camino Churín-Andajes - Valle de Huaura, 2500 msnm.

Categorización y Conservación: Ostolaza et al. (2003) sugieren incluir a este taxón en la categoría de "En Peligro" (Endangered).

Haageocereus acranthus ssp. deflexispinus (Rauh \& Backeberg ) Ostolaza

Distribución: LIMA; Camino Churín-Andajes - Valle de Huaura, 2500 msnm.

Categorización y Conservación: Ostolaza et al. (2003) sugieren incluir a este taxón en la categoría de "En Peligro" (Endangered).

Haageocereus acranthus ssp. olowinskianus (Backeberg) Ostolaza

Distribución: LIMA: Manchay - Valle de Lurín, 200 msnm; Cardal - Valle de Lurín, 230 msnm; Cerros Caracoles, km 55 Pan. Sur, 180 msnm; Cerros Caracoles, km 55 Pan. Sur, 100 msnm.

Categorización y Conservación: Según Ostolaza (2003) este taxón está en la categoría de "Vulnerable" (Vulnerable).

Haageocereus acranthus ssp. olowinskianus forma rubriflorior (Rauh \& Backeberg) Ostolaza

Distribución: LIMA: Cardal - Valle de Lurín, 230 msnm. 
Categorización y Conservación: Según Ostolaza (1996) este taxón debería estar considerado en la categoría de "En Peligro Crítico" (Critically Endangered).

Haageocereus acranthus ssp. olowinskianus forma clavispinus (Rauh \& Backeberg) Ostolaza

Distribución: LIMA; Picapiedra - Valle de Lurín, 200 msnm, Cardal - Valle de Lurín, 230 msnm.

Categorización y Conservación: Según Ostolaza (1996) este taxón debería estar considerado en la categoría de "En Peligro Crítico" (Critically Endangered).

Haageocereus albispinus (Akers) Backeberg

Distribución: LIMA: Valle de Santa Eulalia, 1600 msnm; Valle de Santa Eulalia, Callahuanca, 1300 msnm.

Categorización y Conservación: Por las características de su hábitat, se sugiere ubicar a este taxón en la categoría de "En Peligro" (Endangered).

Haageocereus pseudomelanostele (Werdermann \& Backeberg) Backeberg

Distribución: LIMA: Cajamarquilla, $500 \mathrm{msnm}$; Cajamarquilla, 500 msnm; Quebrada California, 980 msnm.

Categorización y Conservación: Según Ostolaza (2003) este taxón está en la categoría de "En Peligro" (Endangered).

Haageocereus pseudomelanostele ssp. acanthocladus (Rauh \& Backeberg) Ostolaza

Distribución: LIMA: Sayán - Valle de Huaura, 700 msnm.

Categorización y Conservación: Ostolaza et al. (2003) sugieren incluir a este taxón en la categoría de "En Peligro" (Endangered).

Haageocereus pseudomelanostele ssp. carminiflorus (Rauh \& Backeberg) Ostolaza

Distribución: LIMA: Quebrada Tinajas - Valle de Lurín, 570 msnm, y Cerros de Manchay - Valle de Lurín, 200 msnm.

Categorización y Conservación: Según Ostolaza (2003) este taxón está en la categoría de "Vulnerable" (Vulnerable).

Haageocereus pseudomelanoste ssp. setosus (Akers) Backeberg

Distribución: LIMA: Picapiedra - Valle de Lurín, 200 msnm; Cerros Caracoles, km 55 Pan. Sur, 180 msnm; Cerros Caracoles, km 55 Pan. Sur, 100 msnm.

Categorización y Conservación: Según Ostolaza (2003) este taxón está en la categoría de "En Peligro Crítico" (Critically Endangered).

Haageocereus tenuis (Ritter) Rauh \& Ostolaza

Distribución: LIMA; Huacho, km 118 Pan Norte, 300 msnm.

Categorización y Conservación: Por las características del hábitat se sugiere incluir a este taxón en la categoría de "En Peligro Crítico" (Critically Endangered).

XHaagespostoa climaxantha (Werdermann) Rowley
Distribución: LIMA: Quebrada Tinajas - Valle de Lurín, 570 msnm.

Categorización y Conservación: Por las características de su hábitat se sugiere incluir a este taxón en la categoría de "En Peligro Crítico" (Critically Endangered).

XHaagespostoa albisetata (Akers) Rowley

Distribución: LIMA; Valle de Santa Eulalia, 1600 msnm, Valle de Santa Eulalia, Callahuanca, 1300 msnm.

Categorización y Conservación: Por las características de su hábitat se sugiere incluir a este taxón en la categoría de "En Peligro Crítico" (Critically Endangered).

En las Figuras 3 y 4 se muestra la distribución de las especies, subespecies, formas e híbridos estudiados, mientras que en la Tabla 2 las mismas ordenadas según su categoría de conservación. La especie con menor distribución fue $H$. tenuis, la cual, por las características del hábitat se sugiere incluir en la categoría de "En Peligro Crítico", mientras que la especie con mayor distribución fue $H$. acranthus, categorizada como "Vulnerable".

Tabla 2. Categorías de conservación del género Haageocereus e híbridos en el departamento de Lima. Perú.

\begin{tabular}{|c|c|}
\hline $\begin{array}{c}\text { Categoría de } \\
\text { Conservación } \\
(\mathrm{UICN}, 2001)\end{array}$ & $\begin{array}{c}\text { Especies, subespecies, formas e híbridos del } \\
\text { género Haageocereus }\end{array}$ \\
\hline $\begin{array}{l}\text { VULNERABLES } \\
\text { (VU) }\end{array}$ & $\begin{array}{l}\text { H. acranthus } \\
\text { H. acranthus ssp. olowinskianus } \\
\text { H. pseudomelanostele ssp. carminiflorus }\end{array}$ \\
\hline $\begin{array}{l}\text { EN PELIGRO } \\
\text { (EN) }\end{array}$ & $\begin{array}{l}\text { H. acranthus ssp. deflexispinus } \\
\text { H. acranthus ssp. zonatus } \\
\text { H. albispinus } \\
\text { H. pseudomelanostele } \\
\text { H. pseudomelanostele ssp. acanthocladus }\end{array}$ \\
\hline $\begin{array}{l}\text { EN PELIGRO } \\
\text { CRÍTICO } \\
\text { (CR) }\end{array}$ & $\begin{array}{l}\text { H. acranthus ssp. olowinskianus f. clavispinus } \\
\text { H. acranthus ssp. olowinskianus f. rubriflorior } \\
\text { H. pseudomelanostele ssp. setosus } \\
\text { H. tenuis } \\
\text { XHaagespostoa albisetata } \\
\text { XHaagespostoa climaxantha }\end{array}$ \\
\hline
\end{tabular}

Estado de conservación del área de estudio. Todas las localidades presentaron distintos grados de vulnerabilidad, encontrándose más perturbadas aquellas próximas a las periferias de Lima, como son los casos de Cajamarquilla, Santa Clara, Manchay, Cardal, así como Camino a Huacho (km 118 Pan. N.). Para esta última locación, identificada como única área de distribución de Haageocereus tenuis, su proximidad a la carretera y la polución generada por las granjas avícolas circundantes ha ido en desmedro del éxito que tuvo esta especie tiempo atrás. El caso de 


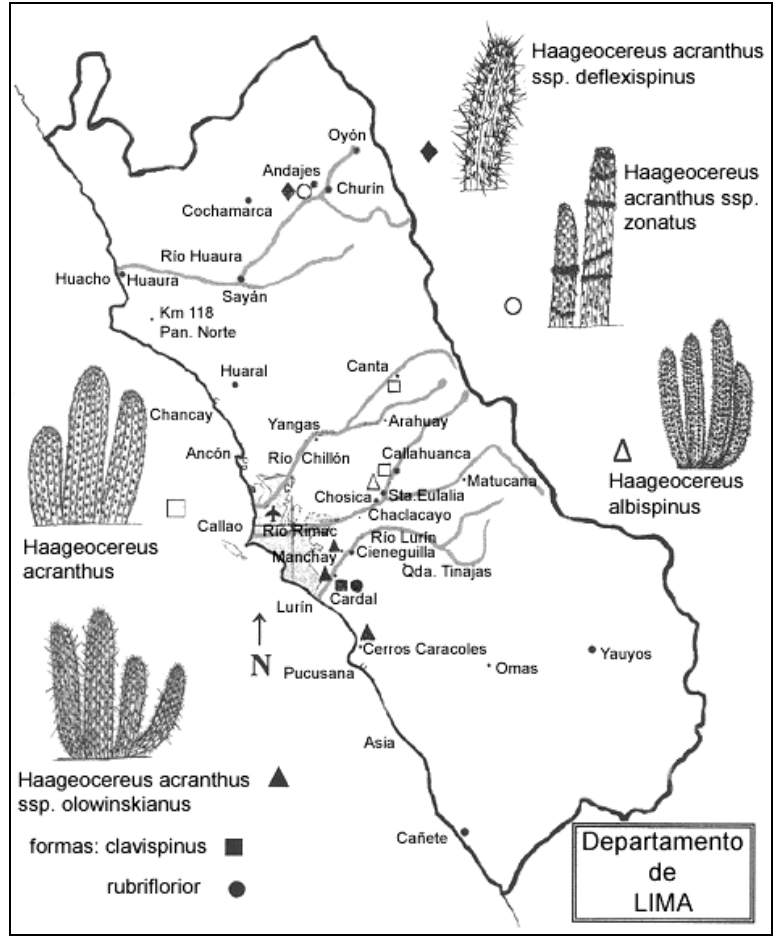

Figura 3. Distribución de las especies, subespecies y formas de Haageocereus acranthus y de H. albispinus en el departamento de Lima. Perú.

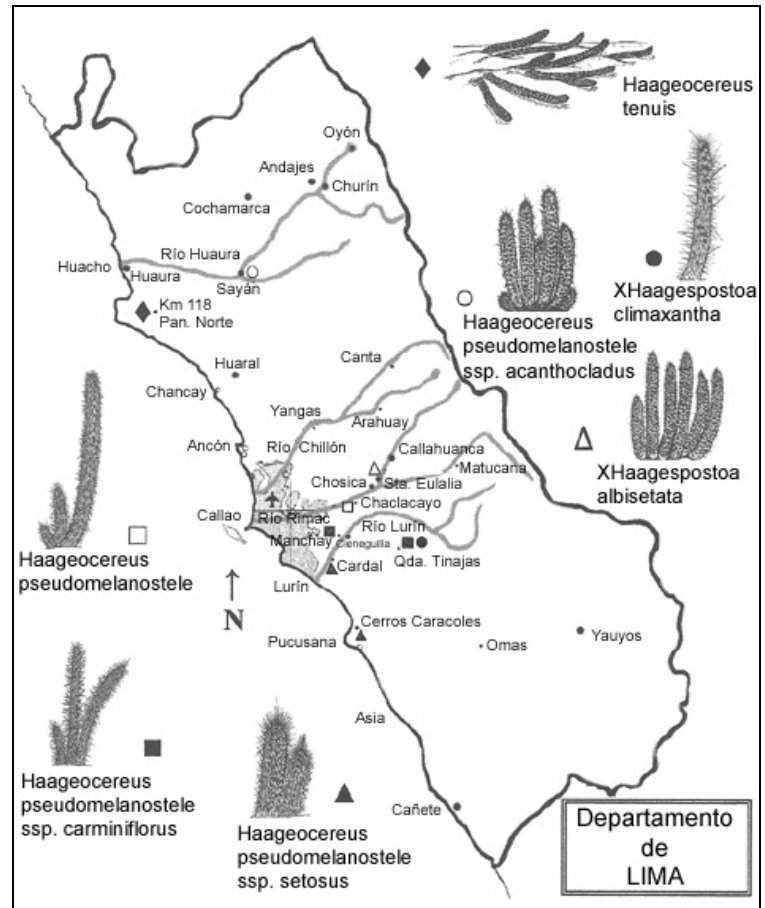

Figura 4. Distribución de las subespecies de Haageocereus pseudomelanostele, de H. tenuis y de los híbridos XHaagespostoa albisetata y XHaagespostoa climaxantha en el departamento de Lima. Perú.

Cajamarquilla es más extremo, en esta localidad se describió la especie tipo del género Haageocereus:
H. pseudomelanostele. Actualmente la expansión urbana ha hecho que ya no se encuentre ningún cactus en la zona. En Santa Clara, se puede apreciar una fase previa al caso de Cajamarquilla, donde la gran mayoría de los cactus replegados en la parte superior del cerro próximo a Santa Clara, están muertos o en un estado deplorable. En Manchay, se observa una situación menos crítica que en Santa Clara, pero de igual vulnerabilidad; los estudios poblacionales en Manchay (Calderón, 2002) muestran densidades de individuos muertos más altas que las densidades de individuos vivos. Los cactus en la localidad de Cardal están circundados por cultivos agrícolas no obstante aún se mantienen en buenas condiciones. Paradójicamente, localidades muy perturbadas del presente estudio o con mayor riesgo de destrucción, como Cajamarquilla, Picapiedra y Cardal, corresponden a zonas arqueológicas declaradas intangibles por el Instituto Nacional de Cultura. La mejor estrategia para la conservación de la diversidad biológica a largo plazo, en estas localidades, sería la conservación de poblaciones y comunidades naturales en su estado silvestre, conocida como conservación in situ (Primack, 1998). Sin embargo, debido a que muchas veces esta opción no resulta viable, por la creciente actividad humana, es probable que el único modo de proteger de la extinción a estas especies sea mediante estrategias de conservación ex situ (Soulé, 1987; Kleiman et al., 1996). En estos casos, los Jardines Botánicos, como centros de conservación ex situ, se encuentran en una posición única de contribuir a los esfuerzos de conservación porque las colecciones vivas en estos centros y su respectivo herbario de colecciones de plantas secas, representan el mejor recurso de información de la distribución y requerimientos de hábitat de las plantas (Primack, 1998).

\section{Conclusiones}

1. Se registraron un total de 12 taxa correspondientes a 4 especies, 6 subespecies y 2 híbridos, además de 2 formas.

2. La especie con menor distribución fue $H$. tenuis, la cual, por las características del hábitat se le ha incluido en la categoría de "En Peligro Crítico", mientras que la especie con mayor distribución fue $H$. acranthus, categorizada como "Vulnerable".

3. Los taxa estudiados se encuentran en las siguientes categorías de conservación: Vulnerable (3), En Peligro (5) y En Peligro Crítico (6).

4. Conocer y reportar la distribución de las especies estudiadas es vital para iniciar los planes de conservación de las mismas, así como de sus hábitats.

5. El caso más extremo de deterioro de hábitat es la localidad de Cajamarquilla, donde fue descrita la especie tipo del género, $H$. pseudomelanostele. 
Actualmente ya no se encuentra ninguna especie de cactus en la zona. Mientras que, entre los hábitats seriamente perturbados, cuyo estado de conservación aún no es tan crítico, están Quebrada Tinajas, Santa Eulalia y El Chillón (Altura km 69 C. Lima-Canta), sobre los cuales se pueden proponer estrategias de conservación in situ.

6. Los planes de conservación ex situ constituyen una alternativa importante y quizás única para aquellas especies que han perdido su ambiente natural o que se encuentran en Peligro Crítico.

\section{Agradecimientos}

Los autores expresan su agradecimiento al Blgo. Gustavo Dreyfus, Estudiantes Sidney Novoa, Francis van Oordt y Michael Vega, por su apoyo en el trabajo de campo y de gabinete y a todos los alumnos del Curriculum de Biología que han participado en el Proyecto de Cactáceas de Lima y Cactario del Jardín Botánico “Octavio Velarde Núñez” de la Universidad Nacional Agraria La Molina. Asimismo, al Fondo Especial de Desarrollo Universitario (FEDU) y a la Sociedad Británica de Cactus y Suculentas por el apoyo financiero.

\section{Literatura citada}

Akers J. 1947. New Genus and New Species from Perú. Cactus \& Succulent Society of America. XIX-5: 67-70.

Arakaki M. 2000. Manual for Collection Techniques, Preparation and Management of Cactaceae Specimens. Natural History Museum Science (BIO 371 G) University of Texas Herbarium (TEX).

Backeberg C. 1960. Die Cactaceae. Tomo II: 1159 1245.

Brako L. \& Zarucchi J.L. 1993.Catálogo de las Angiospermas y Gimnospermas del Perú. Missouri Botanical Garden.

Britton N.L. \& Rose J.N. 1919-1922. The Cactaceae, 4 vols. Carnegie Intitution. Washington D.C.

Calderón N. 2002. Diagnóstico Poblacional de las cactáceas de Manchay, Prov. de Lima. Quepo 16: 40-45.

2003. Estudio Taxonómico del género Haageocereus (Familia Cactaceae) en el Departamento de Lima. Tesis. Facultad de Ciencias. Universidad Nacional Agraria La Molina.

Eggli U. \& Taylor N. 1990. IOS INDEX of names of Cactaceae published 1950-1990 from Repertorium Plantarum Succulentarum. Royal Botanic Gardens of Kew; Int. Organization for Succ. Plant Study; and Städtische Sukkulenten-Sammlung Zürich.

Hunt D. 1999. Cites Cactaceae Checklist. 2da edición. $315 \mathrm{pp}$.

Instituto Nacional de Recursos Naturales (INRENA). 1995. Mapa Ecológico del Perú. Guía explicativa del Mapa Ecológico del Perú.

Kleiman D., Allen M., Thompsom K. \& Lumpkin S. 1996. Wild Animals in Captivity: Principles and Techniques. University of Chicago Press, Chicago.

Ostolaza C. 1980. Las cactáceas y la conservación de la flora. Boletín de Lima. 7: 40-44. Lima

1988. Los cactus de los alrededores de la ciudad de Lima y su conservación. Zonas Aridas. No 5. UNALM. Lima

1995. Cacti in the vicinity of Lima- Peru and their conservation status. Cac. \& Succ. Journal. 67 (1).

1996. A Closer Look at the Conservation Status of Cacti in the Vicinity of Lima, Perú. British Cactus \& Succulent Journal. 14 (4):158.

1998. Las cactáceas y la conservación de la flora. Boletín de Lima. No 7. Edit. Los Pinos. Lima.

2002. Cactus de Lima en peligro de extinción. Quepo. Lima. 16: 56-58. 2003. Categorización y Conservación de Cactáceas Peruanas. Zonas Aridas. (7): 147-159.

Ostolaza C., Ceroni A., Dreyfus G., Calderón N. \& Novoa S. 2003. Cacti of the Huaura river valley, Perú - a conservation study. British Cactus \& Succulent Journal. 21 (2): 87-97.

Primack R.B. 1998. Essentials of Conservation Biology. Segunda Edición. Boston University.

Rauh W. 1958. Beitrag zur Kenntnis der perunischen Kakteenvegetation V. Zitzungsberichte fur Heidelberger Akademie der Wissennschaften, Botanisches Institut der Universitat Heidelberg Heidelberg. Springer-Verlag. : 368-435.

Ritter F. 1980. Kakteen in Südamerika. Band 3. F. Ritter Sebbstverlag, Spangenberg.

Soulé M. 1987. Viable Populations for Conservation. Cambridge University Press, Cambridge, UK.

Unión Internacional para la Conservación de la Naturaleza. 2001. UICN Red List Categories, versión 3.1. Gland, Switzerland.

Weberbauer A. 1945. El Mundo Vegetal de los Andes Peruanos. Estudio Fitogeográfico. 2da Edición. Estación Experimental Agrícola de La Molina. Dirección de Agricultura. Ministerio de Agricultura. Lima.

\footnotetext{
${ }^{1}$ Jardín Botánico “Octavio Velarde Núñez”, Universidad Nacional Agraria La Molina. natcalderon@yahoo.com / aceroni@lamolina.edu.pe, Apartado postal: 12-056., Lima 12, Perú.

${ }^{2}$ Sociedad Peruana de Cactus y Suculentas. carlosto@ec-red.com, apartado postal 3215, Lima 100. Perú. Fax (511)4792630.
} 